\title{
Frictional heterogeneities can promote disordered slip evolution on faults
}

\author{
Sohom Ray \\ Civil and Environmental Engineering, Tufts University, Medford, MA 02155, USA \\ Sohom.Ray@tufts.edu
}

3 September 2019

\begin{abstract}
We consider that a slip instability nucleates an earthquake. Past studies found blow-up solutions for diverging slip velocities. Prior stability analyses, considering heterogeneous frictional properties revealed that stable blow-up solutions can predictably dictate earthquake-nucleating instabilities. In this prior analysis, the focus remained mainly on the attraction to stable blow-up solutions. Here, we shift the focus of discussion towards unstable blow-up solutions, in particular, we seek frictional heterogeneities that can make all blowup solutions to lose stability. (We consider variations in direct and evolution effect parameters, $a(x)$ and $b(x)$, and pressume that $D_{c}$ and $\sigma$ are uniform). We find that faults that include rate-strengthening regions can lack attractive blow-up solutions. In such scenarios, a rate-weakening fault that includes appropriate ratestrengthening regions can significantly delay the development of an instability compared to a fault that is entirely rate-weakening. That delay can be attributed to the loss of stability of the blow-up solutions. Owed to the non-existence of attractive blow-up solutions, the developing slip velocity fails to converge to a specific distribution at a single location. The near-chaotic transient dynamics may give rise to tremor-like activities.
\end{abstract}

\section{KEYWORDS}

Slip instabilities. Dynamical System. Blow-up solutions. Fixed point iterations. Elasto-frictional lengthscale. Frictional heterogeneities. Stability analysis.

\section{INTRODUCTION}

The slip on model faults evolves under two basic physical ingredients: interfacial friction and host-rock's elasticity. Their coupling modifies the available length or slip scale, for example, slip-weakening distance or fault roughness, to produce elasto-frictional length: a characteristic length for fault slip evolution. An earthquake nucleates when inertia limits the continued acceleration of slip and drives an outward propagating dynamic rupture. Here, we neglect the inertia which, consequently, prevents dynamic rupture and leads to unbounded 
growth of slip rate: an instability provokes an earthquake and its analysis resolves the relevant length and time scales.

Like instabilities in nature, which results from differential activities (e.g., Weber et al., 2018), an earthquake nucleating slip instabilities are owed to the larger weakening than strengthening of fault's frictional strength in reply to velocity increases. We consider a rate- and state-dependent formulation of fault's frictional strength (Dieterich, 1978a; Ruina, 1983) (see section 1 of the supplementary information)

$$
f(x, t)=f_{o}+a \ln \left[\frac{V(x, t)}{V_{o}}\right]+b \ln \left[\frac{\theta(x, t)}{D_{c} / V_{o}}\right]
$$

where $V(x, t)=\partial \delta / \partial t$ is the velocity of slip and $\theta(x, t)$ is the conventional state variable, and $D_{c}$ is the slip scale over which friction attains a steady-state. In addition, we express the state or history of slip through a surrogate state variable $\Phi(x, t)$ defined using the following relation

$$
\partial_{t} \theta=-\frac{V(x, t) \theta(x, t)}{D_{c}} \Phi(x, t)
$$

For the aging-law of state evolution $\Phi(x, t)$ is given by,

$$
\Phi(x, t)=1-\frac{D_{c}}{V(x, t) \theta(x, t)} .
$$

and for slip-law of state evolution, $\Phi(x, t)=\ln \left(V(x, t) \theta(x, t) / D_{c}\right)$.

$\Phi(x, t)$ is a convenient measure for nearness of fault slip to steady-state sliding: $\Phi \approx 0$ corresponds to steady-state sliding wherein the behavior of slip-law is indistinguishable from aging-law. Far from the steadystate $\Phi=1$ for aging-law; and differs from slip-law by a factor of $\ln \left(V \theta / D_{c}\right)$. When considered variation of frictional properties stay close to rate-neutral region, $\Phi(x, t)$ is expected to remain very close to zero in most part of the fault and consequently the slip evolution remains independent of the chosen evolution law.

In steady state, the fault friction is rate dependent

$$
f_{s s}(x, t)=f_{o}-(b-a) \ln \left[\frac{V(x, t)}{V_{o}}\right]
$$

and the contrast between the weakening-coefficient, $b$, and the instantaneous strengthening-coefficient, $a$, dictates the manner of slip evolution on faults.

When magnitude of strengthening exceeds that of weakening, that is with $b<a$, a slip evolve aseismically and any transient acceleration decays over time. On the contrary, when fault's weakening dominates, that is $b>>a$, a transient acceleration manifest into an instability over a finite region. However, the tendency of slip to manifest into an instability decreases as weakening becomes less dominant. This is apparent from the progressively larger support length required to nucleate an instability when $b$ approaches $a$. The two parameter regimes of disparate slip development — aseismic and potentially seismic — are mediated through an infinite nucleation length at $b=a$ (e.g., Rubin \& Ampuero, 2005; Viesca, 2016b; Garagash \& Germanovich, 2012).

When $b$ marginally exceeds $a$, the slip instabilities on homogenous model faults manifest on large nucleation lengths and do so chaotically (e.g., Viesca, 2016b; Rubin \& Ampuero, 2005). We find that a fault's frictional heterogeneity that includes appropriate rate-strengthening regions could also lead to a spatially distributed near-chaotic slip evolution, however, no large nucleation length is required to do so. Here, by near-chaotic we refer to the apparently unpredictable slip development at different regions of the fault. However, two nearly same initial conditions do not show large deviation in the ultimate slip development. We find 
that including such heterogeneity makes the fault devoid of any asymptotically stable blow-up solution and can create tremor bursts.

In what follows we briefly review the conditions of slip instabilities on fault. We describe the methods to solve for the blow-up solutions on model faults of two disparate elastic configurations: slip surfaces parallel to an overriding thin slab, and between two half-spaces. Then we briefly review the stability regimes of fixed point solutions of Viesca (2016b) followed by a short analysis of heterogeneity within those regimes. Subsequently, we shift our attention to unstable blow-up solutions and explore the fault conditions that might encourage the blow-up solutions to lose stabilities.

\section{CONDITIONS OF SLIP RATE INSTABILITY}

In this section we assume frictional parameters to be uniform and re-express the slip rate and state evolution of Ray \& Viesca $(2017,2019)$ using two non-dimensional operators $\mathcal{V}$ and $\mathcal{S}$, respectively, for slip velocity and state. For the purpose of this section, we non-dimensionalize time and velocity using substitutions $t \rightarrow$ $(b / a)\left(D_{c} / V_{o}\right) t, V \rightarrow V_{o} v$, and consider frictional parameters to be uniform along the fault. The dimensionless velocity and state evolution are, succinctly, given by,

$$
\begin{aligned}
\partial_{t} v & =v \mathcal{V} v \\
\partial_{t} \Phi & =(1-\Phi) \mathcal{S} v
\end{aligned}
$$

where the operators $\mathcal{V}=\left(D_{c} / b \sigma\right) \mathcal{L}+\Phi(x, t)$ and $\mathcal{S}=\left(D_{c} / b \sigma\right) \mathcal{L}+r \Phi(x, t)$, where $r=(b-a) / b$ and $\mathcal{L}$ is the traction-slip operator. Here, we have suppressed an explicit depedendnce of the physical quantities on position and time.

For thin slab elastic configuration $\mathcal{L}=\bar{E} h \partial_{x x}^{2}$ where the elastic modulus $\bar{E}=2 \mu /(1-\nu)$ and $\bar{E}=\mu$ for mode-II and mode-III sliding respectively. $\mu$ and $\nu$ are the shear modulus and Poisson's ratio, respectively. The dimensionless operators for slip rate and state, for thin slab elastic configurations, are given by

$$
\begin{aligned}
& \mathcal{V}=L_{b h}^{2} \partial_{x x}^{2}+\Phi(x, t) \\
& \mathcal{S}=L_{b h}^{2} \partial_{x x}^{2}+r \Phi(x, t)
\end{aligned}
$$

where $L_{b h}=\sqrt{\bar{E} h D_{c} /(b \sigma)}$ is the relevant elastofrictional length-scale for thin-slab type elastic configuration with homogenous $b$. An analysis of the evolution equation (4), just before the late-stage non-linearity starts to dominate, is equivalent to the analysis of the above operators. Their spectrum (eigenvalues) dictate if perturbations grow or shrink in amplitude. We consider monochromatic perturbation to slip rate of the form $v(x, t)=1+\cos \left(\frac{2 \pi}{\lambda} x\right)$. The eigenvalue of least magnitude of the Lapalcian, $\partial_{x x}^{2}$, under Neuman boundary conditions is equal to $-4 \pi^{2} / \lambda^{2}$. On substituting this eigenvalue in above, $\partial_{x x}^{2} \rightarrow-4 \pi^{2} / \lambda^{2}$, and imposing a characteristic value, $\Phi_{o}$, for the surrogate state variable, yields the relevant eigenvalues for the velocity and state operators. Which are, respectively, given by,

$$
\begin{aligned}
& \Lambda_{v}=-\frac{4 \pi^{2} L_{b h}^{2}}{\lambda^{2}}+\Phi_{o} \\
& \Lambda_{s}=-\frac{4 \pi^{2} L_{b h}^{2}}{\lambda^{2}}+r \Phi_{o}
\end{aligned}
$$


A perturbation can grow in amplitude when above eigenvalues are positive. We now find the conditions that can ensure they remain so. If steady state sliding is imposed, $\Phi(x, t)=0$, the eigenvalues $\Lambda_{s}$ and $\Lambda_{v}$ are negative for all the wavelengths, consequently, the perturbations decay with time. Which is to say that instabilities will not manifest unless slip is little above steady-state sliding. A positive $\Lambda_{v}$ is required for velocity perturbations to grow in amplitude, which, in turn, requires $\Phi_{o}$ to be positive. Both the conditions are fulfilled and a monochromatic perturbation is guaranteed to grow in magnitude when

$$
\Lambda_{s}>0
$$

which is equivalent to

$$
\frac{4 \pi^{2} L_{b h}^{2}}{\lambda^{2}}<r \Phi_{o}<r
$$

Because $\Phi_{o}$ is unknown a priori, we use its upper bound to get the critical wavelength for the monochromatic perturbation to grow with time, given by,

$$
\lambda>\frac{2 \pi L_{b h}}{\sqrt{1-a / b}}=\lambda_{c r}
$$

When nonlinear effects dominate the actual size over which an instability ultimately develops, is considerably different form the critical wavelength and scales as $\lambda_{c r} \Phi_{o}^{-1 / 2}$.

We note that when slip evolves far from steady-state (i.e., $\Phi(x, t)=1$ ), equation (4) does not participate. In this case, the critical wavelength is given by obtained by $\Lambda_{v}>0$ which is equal to $2 \pi L_{b h}$. We note that the critical wavelength could be very close to or disparate from the elasto-frictional lengthscales depending upon the extent of strengthening-weakening contrast.

\section{NONLINEAR ANALYSIS OF SLIP}

We move beyond the linear regime and analyze fault slip when nonlinearity dominates. A positive $\Lambda_{v}$ makes sure that corresponding mode increase with time such that the (dimensionless) slip accelerates as $\partial_{t} v \sim \Lambda_{v} v^{2}$ leading to a blow-up in finite-time $t=t_{i n}$. Owed to the singularity at $t=t_{i n}$, a numerical integration method requires an ever-increasing precision to continue evolving slip rate and state near the instability time and most common methods eventually fails. Consequently, this also prevents the full resolution of the length scales (or the support) over which the instability manifests. This limitation is particularly detrimental when fault frictional properties are variable in space: in response to an initial perturbation, slip rate is expected to migrate to frictionally favorable regions, which, in turn, requires numerical integrations to be carried out to the time of instability, $t=t_{i n}$, and to very high slip rates.

An alternate route overcomes those numerical challenges and, instead, looks for the similarity solutions of the form (e.g., Rubin \& Ampuero, 2005; Viesca, 2016b,a)

$$
V(x, t)=\frac{D_{c}}{t_{f}(t)} W(x, t)
$$

where $t_{f}(t):=t_{\text {in }}-t$, is the relevant time scale at the late stage of the slip velocity evolution. Substituting the 
above ansatz in (4), we get

$$
\begin{aligned}
t_{f} \partial_{t} W & =W[\mathcal{V} W-a / b] \\
t_{f} \partial_{t} \Phi & =(1-\Phi) \mathcal{S} W .
\end{aligned}
$$

(for the sake of notational convenience, here we suppress an explicit dependence of frictional paremeters on position).

When the instability is approached, $t_{f} \rightarrow 0$, the left side approaches a steady-state only if $\partial_{t} W\left(\right.$ and $\left.\partial_{t} \Phi\right)$ diverges at a rate slower than $O\left(t_{f}^{-1}\right)$. That is, $\partial_{t} W$ (and $\partial_{t} \Phi$ ) scales as $O\left(t_{f}^{-1+\epsilon}\right.$ ) where $\epsilon>0$. We presume this the case and the system (10) reaches a steady-state. We go ahead in time and find the equilibrium solutions (not a mechanical equilibrium) at $t=t_{i n}: W\left(x, t=t_{\text {in }}\right) \rightarrow \mathcal{W}(x)$ and $\Phi\left(x, t=t_{i n}\right) \rightarrow \mathcal{P}(x)$ as $t \rightarrow t_{\text {in }}$. We re-express the presumption using the following notation:

$$
t_{f} \frac{\partial}{\partial t}\left[\begin{array}{c}
W \\
\Phi
\end{array}\right]=0 \Longrightarrow W\left(x, t \rightarrow t_{i n}\right) \rightarrow \mathcal{W}(x) \quad \text { and } \quad \Phi\left(x, t \rightarrow t_{i n}\right) \rightarrow \mathcal{P}(x)
$$

where we note that the vice-versa need not to be true (next section).

The spatial profiles $\mathcal{W}(x)$ and $\mathcal{P}(x)$, are given by,

$$
\begin{aligned}
& 0=\mathcal{V} \mathcal{W}-a / b \\
& 0=[1-\mathcal{P}][1-\mathcal{W} \mathcal{P}]
\end{aligned}
$$

where the last equation uses $\mathcal{S} \mathcal{W}=[1-\mathcal{W} \mathcal{P}](a / b)$ and the velocity operator $\mathcal{V}=L_{b h}^{2} \partial_{x x}^{2}+\mathcal{P}(x)$, where, $\mathcal{W}(x) \mathcal{P}(x)$ is either unity or $\mathcal{W}(x)$ depending upon whether $\mathcal{W}$ is greater or lesser than unity (Viesca, 2016b,a; Ray \& Viesca, 2017, 2019). Therefore, the blow-up solution for diverging slip rate is given by

$$
V(x, t)=\frac{D_{c}}{t_{f}(t)} \mathcal{W}(x)
$$

which Rubin \& Ampuero (2005) and Viesca (2016a,b) found to exist for homogeneous faults and Ray \& Viesca $(2017,2019)$ for heterogeneous ones.

\subsection{BLOW-UP SOLUTIONS FOR HALF-SPACE ELASTIC CONFIGURATION}

In case of slip surface within two elastic half-spaces the problem (12a) reduces to an integro-differential equation with distribution $\left.\mathcal{W}(x), l_{1}\right]$ and $l_{2}$, are unknowns. We note that the coordinate $x$ in this case is expressed in the units of $L_{b}$ (and not $L_{b h}$ ). The system of equations to solve for is given by

$$
\begin{aligned}
& \frac{1}{2 \pi} \int_{l_{1}}^{l_{2}} \frac{d \mathcal{W}(y) / d y}{y-x} d y+\mathcal{W}(x) \mathcal{P}(x)-\frac{a(x)}{b}=0 \\
& \mathcal{W}\left(l_{1}\right)=0, \mathcal{W}\left(l_{2}\right)=0 \\
& \mathcal{W}^{\prime}\left(l_{1}\right)=0, \mathcal{W}^{\prime}\left(l_{2}\right)=0
\end{aligned}
$$

wherein the distribution $\mathcal{W}(x)$, and the boundaries $l_{1}$ and $l_{2}$ are to be determined. We note that the function $\mathcal{W}(x) \mathcal{P}(x)$ is equal to either 1 or $\mathcal{W}(x)$ depending upon whether $\mathcal{W}(x)$ is greater or lesser than unity. In above we interpret $x$ in the units of the elasto-frictional lengthscale $L_{b}$. 
In case of homogeneous properties, the problem simplifies to a symmetric distribution $\mathcal{W}(x)$ and its support with half-length $l=l_{2}=-l_{1}$. We note that multiple pairs $(\mathcal{W}(x), l)$ exist that satisfy the above equation. However, our interest lies on the blow-up solutions (13) with minimum support length: larger support length solutions are unattainable. Likewise, under heterogenous conditions, multiple triplets $\left(\mathcal{W}(x), l_{1}, l_{2}\right)$ satisfy the above equations and we look for the solutions where $\mathcal{W}(x)$ remains nonzero within $l_{1}$ and $l_{2}$.

To find them, the problem (12a) is discretized into a set of non-linear algebraic equations and the solution found by iterative Newton-Raphson root finding. The convergence to the desired solution under nonuniform $a(x) / b$ is complicated owed to broken translational invariance: that is, multiple solutions for $\mathcal{W}(x)$ exist at different locations in space.

To ensure convergence of the Newton-Raphson iterations, we recast the problem (14) in a translating coordinates, that is, using the variable transformation $x=l \bar{x}+c$ where $\bar{x} \equiv[-1,1], l=\frac{1}{2}\left(l_{2}-l_{1}\right)$, and $c=\frac{1}{2}\left(l_{1}+l_{2}\right)$, and look for the solutions of the form

$$
\begin{aligned}
& \mathcal{W}(x)=u\left(\frac{x-c}{l}\right)=u(\bar{x}) \\
& \mathcal{P}(x)=p\left(\frac{x-c}{l}\right)=p(\bar{x})
\end{aligned}
$$

In the new coordinates, the above problem reduces to the following system of equations:

$$
\begin{aligned}
& U[u(\bar{x}), l, c] \equiv \frac{1}{2 \pi l} \int_{-1}^{1} \frac{d u(\bar{y}) / d \bar{y}}{\bar{y}-\bar{x}} d \bar{y}+u(\bar{x}) p(\bar{x})-\frac{a(l \bar{x}+c)}{b}=0, \\
& \text { with } u(-1)=0 \text { and } u(1)=0 \\
& \left.L[u(\bar{x}), l, c] \equiv \frac{d u(\bar{x})}{d \bar{x}}\right|_{\bar{x}=-1}=0, \\
& \left.C[u(\bar{x}), l, c] \equiv \frac{d u(\bar{x})}{d \bar{x}}\right|_{\bar{x}=+1}=0 .
\end{aligned}
$$

where we note that the function $u(\bar{x}) p(\bar{x})$ is equal to either 1 or $u(\bar{x})$ depending upon whether $u(\bar{x})$ is greater or lesser than unity. A Chebyshev discretization, evaluation of the quadrature, and the Newton-Raphson implementation are discussed in the supplementary meterials.

\section{STABILITY ANALYSES OF THE BLOW-UP SOLUTIONS}

Once the distributions $u(\bar{x})$, and length of the support and location of the peak, $l$ and $c$, respectively, are solved, we map them back to the physical coordinates using (15) to get the blow-up solutions- $\mathcal{W}(x)$ and its extent $l_{1}$ and $l_{2}$.

The solutions $\left(\mathcal{W}(x), l_{1}, l_{2}\right)$ require a posterior stability analysis to ensure if the triplet $\left(\mathcal{W}(x), l_{1}, l_{2}\right)$ satisfies the presumption (11). To do so, a change of the independent variable and the presumption (11) suggests a transformation of the form:

$$
t_{f} \partial_{t} \rightarrow \partial_{s}
$$

and $W(x, t) \rightarrow W(x, s(t))$ where $s$ is a slowed-time variable, given by,

$$
s:=-\ln \left(t_{f} / t_{o}\right)
$$




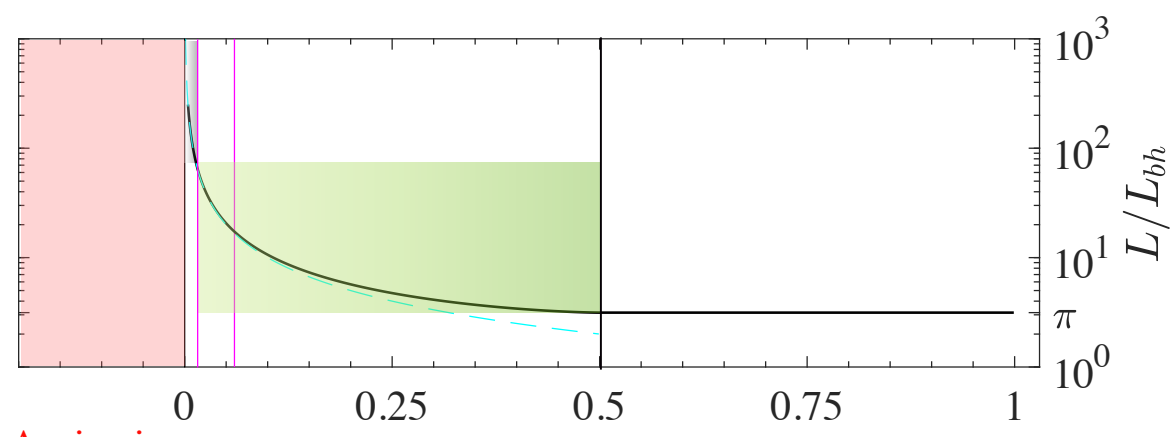

Aseismic

Chaos

Transition regime
Asymptotically stable

Hopf bifurcations

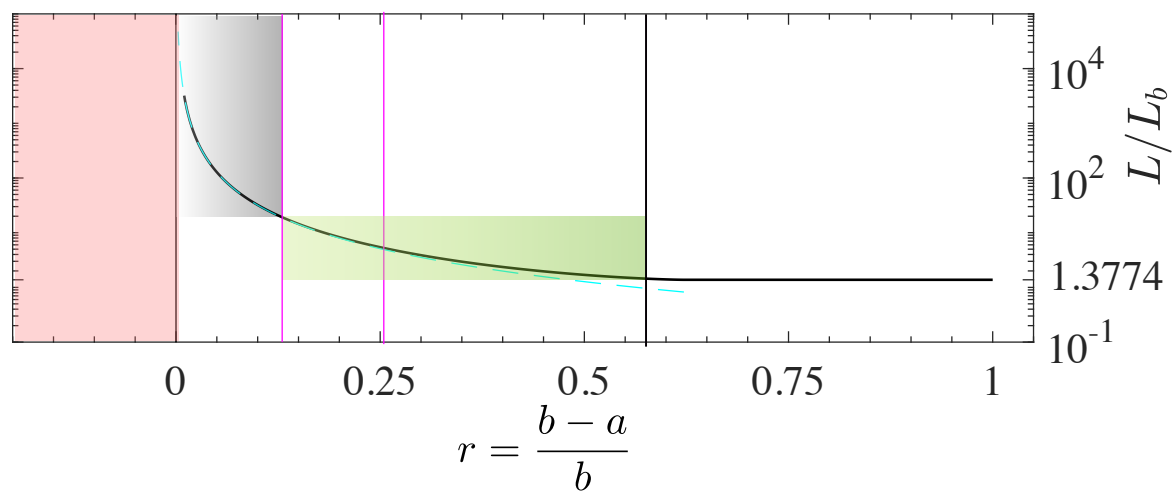

Figure 1. Stability regimes of the blow-up solutions and nucleation length for slip surfaces below a thin slab (top) and within half-spaces (bottom): For large values of $r$, the blow-up solutions are asymptotically stable and the support length remains independent of $r$ as pointed out by Viesca (2016b,a) which coincides with Rubin \& Ampuero (2005) simulations for unstable accelerations in the no-healing regime. In the intermediate range of $r$, the support length increases with decreasing $r$; and the the blow-up solutions show progressively stronger unstable mode with decreasing $r$. We refer this range of $r$ as transition regime wherein the blow-up solutions transitions from an asymptotically stable to a completely chaotic state through series of Hopf bifurcations (Viesca, 2016b,a). The support lengths, for local and nonlocal type of elastic configurations, respectively, scale as $L / L_{b h} \sim r^{-1}$ and $L / L_{b} \sim r^{-2}$ with decreasing $r$. The nucleation length within the transition regime modestly deviates from the fixed-length solution till the onset of the third Hopf bifurcations. However, from the end of the transition and in the chaotic regimes the nucleation size increases by orders of magnitude and diverge as $L / L_{b h} \sim r^{-1}$ and $L / L_{b} \sim r^{-2}$, respectively, with $r \rightarrow 0$ from above. Transition to aseismic regime is mediated through an infinite nucleation length (e.g., Garagash \& Germanovich, 2012) and completely chaotic blow-up solutions.

where $t_{o}$ is any time scale, and instability $t_{f} \rightarrow 0$ now corresponds to $s \rightarrow \infty$. The above change of variable -from normal time scale $\left(t_{f}:=t_{i n}-t\right)$ to a logarithmically stretched one -is apparent from the left side of the equations (10) and (11). That is,

$$
t_{f} \frac{\partial}{\partial t}=-\frac{\partial}{\partial t_{f} / t_{f}}:=\frac{\partial}{\partial s}
$$

(In above, we switched to the traditional symbol for partial derivatives for clarity). 
The evolution for profiles of slip rate and state with the new independent variable, given by,

$$
\begin{aligned}
\partial_{s} W & =W[\mathcal{V} W-a / b] . \\
\partial_{s} \Phi & =(1-\Phi) \mathcal{S} W .
\end{aligned}
$$

constitutes a dissipative dynamical system. When the dependent variables $W(x, s)$ and $\Phi(x, s)$, when $s \rightarrow$ $\infty$, attain $s$-independent steady-state profiles those solutions $\mathcal{W}(x)$ and $\mathcal{P}(x)$ are referred to as fixed point solutions. Those profiles are obtained by equating left side to zero, which, in turn, yields (12a) implying blowup solutions $(\mathcal{W}(x), \mathcal{P}(x))$ are the fixed points of the (dissipative) dynamical system (e.g., Viesca, 2016a,b; Ray \& Viesca, 2017, 2019).

We linearize the dynamical system about the fixed points $(\mathcal{W}, \mathcal{P})$ and analyze the spectrum of the resulting Jacobian (matrix when discretized). The eigenvalues of the Jacobian, given below, dictates the stability of the blow-up solutions.

$$
J=\left[\begin{array}{cc}
\mathcal{W} \mathcal{V} & \mathcal{W}^{2} \\
(1-\mathcal{P}) \mathcal{S} & -\mathcal{S} \mathcal{W}+r(1-\mathcal{P}) \mathcal{W}
\end{array}\right]
$$

The stability of the blow-up solutions is determined by the eigenvalue with a largest real part (other than the symmetry modes described below). We distinguish that eigenvalue using the notation $\lambda_{\max }$. If a mode exists with $\operatorname{Re}\left(\lambda_{\max }\right)>0$, then the solution (13) is said to be unstable, and the solution is stable in the absence of such a mode.

We note that eigenvalues $\lambda=0$ and $\lambda=1$-with respective eigenfunctions $\mathcal{W}^{\prime}(x)$ and $\mathcal{W}(x)$-correspond to, respectively, spatial and temporal symmetry of the problem: they have no bearing on the stability of the blow-up solutions (e.g., Viesca, 2016a; Ray \& Viesca, 2017, 2019). Temporal invariance prevails for all problem parameters, and, in turn, the temporal symmetry mode appears in the stability analysis of the solutions. However, the spatial symmetry mode $(\lambda=0)$ appears when all the problem parameters remain uniform in space.

\subsection{Regimes within rate-weakening}

Following Viesca (2016b,a), in Figure 1 we briefly summarize the dependence of the stability of the blow-up solutions with the parameter $r=1-a / b$. We recall that $r$ denotes the contrast of weakening and strengthening magnitudes of fault friction, relative to weakening magnitude. Large positive values of $r$-significantly dominant weakening than fault's strengthening — promote instabilities as also reflected from the attractive nature of the blow-up solutions in the large $r$ regime. The tendency of slip to diverge gradually diminishes with shrinking $r$ as marked by emergence of increasing strength and number of unstable eigenmodes of the blow-up solutions.

The fixed point solutions (13) are asymptotically stable when fault's weakening considerably exceeds its strengthening. In homogeneous case, the blow-up solutions (13) on a thin-slab elastic configuration are the asymptotically stable when fault weakens with slip rate by, at least, twice it strengthens: $b>2 a$. However, for slip surfaces within half-spaces, the asymptotic stability requires further greater magnitude of weakening: $b>2.6448 a$. An interesting avenue for future work can be to explore why and how those numeric pre 


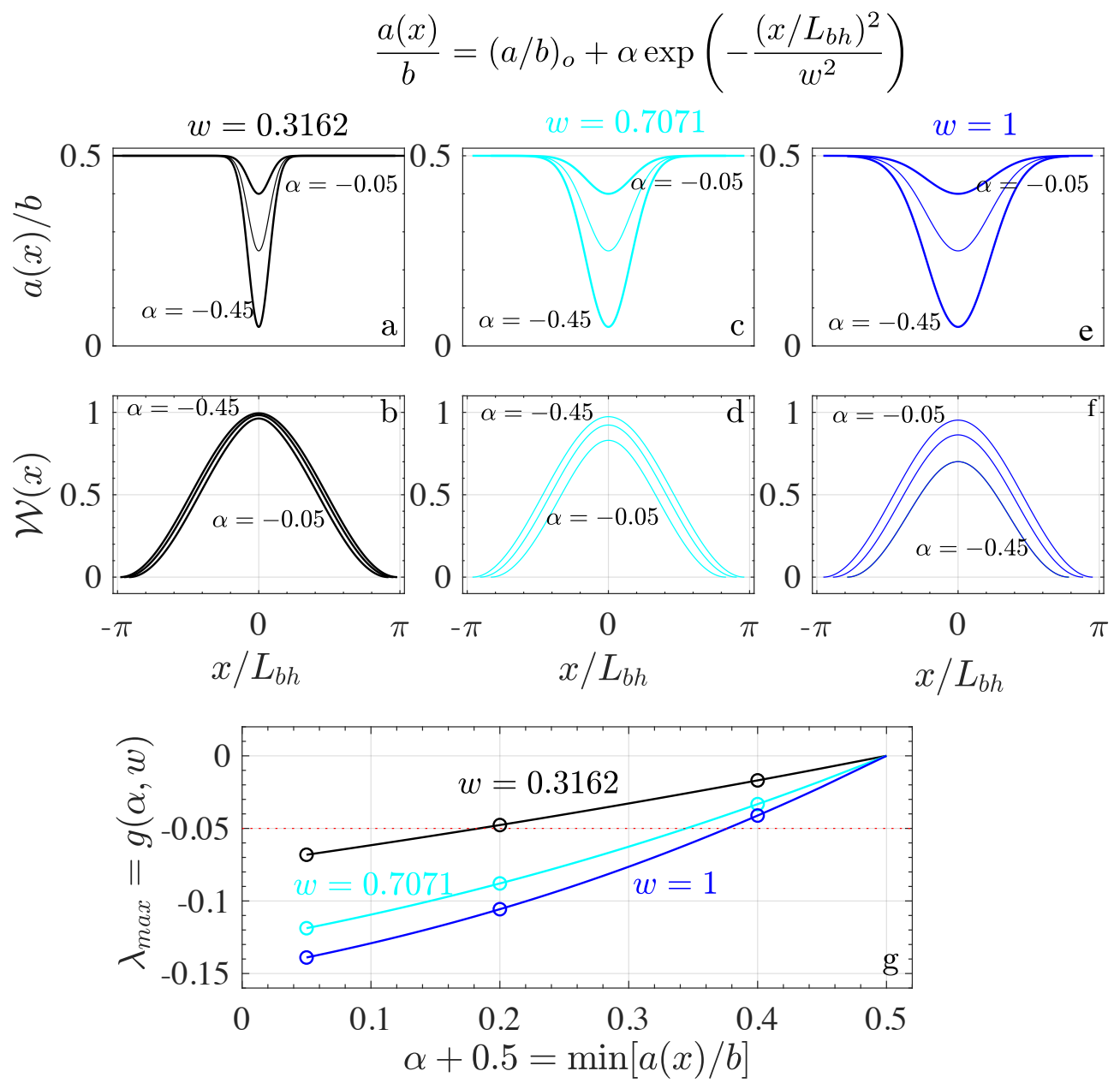

Figure 2. Blow-up solutions under different heterogeneities with with similar stability: (a,c,e) We consider heterogeneities of the form $a(x) / b=(a / b)_{o}+\alpha \exp \left(-\frac{x^{2} / L_{b h}^{2}}{w^{2}}\right)$, where $w$ is the width of the Gaussian and $(a / b)_{o}=0.5$ is the homogeneous reference. The choice $(a / b)_{o}=0.5$, instead of a larger value, keeps the solutions within the asymptotic stability regime. The corresponding blow-up solution is shown in (b,d,f). (g) Stability of the blow-up solutions as a function of the trough $\left((a / b)_{o}-\alpha\right)$ and the width $w$. The circles on the plot correspond to the solutions in $(\mathbf{b}, \mathrm{d}, \mathbf{f})$. A horizontal dashed line, for example the dashed red line, indicates that different heterogeneities have identical stability.

factors depend on the chosen elastic configuration. For heterogeneities within each regime of the parameter the blow-up solutions come in pairs: asymptotically stable and unstable solutions discretely distributed on the fault. Which yields local stability separations and can be attributed as a consequence of the broken translational invariance in space.

In a different regime of parameter space where fault's weakening only marginally exceeds its strengthening, the dynamical system (18) exhibits a chaos. The solutions of the type (13) exist in this range as well; however, they remain unstable, owed to the large number of Hopf bifurcations of eigenmodes, which ultimately results in a chaotic evolution of slip-rate (Viesca, 2016b,a). Such chaotic evolution occurs when weakening is only slightly greater compared to the strengthening: $b / a \approx 1.01$ and 1.14 , respectively, for local and non- 


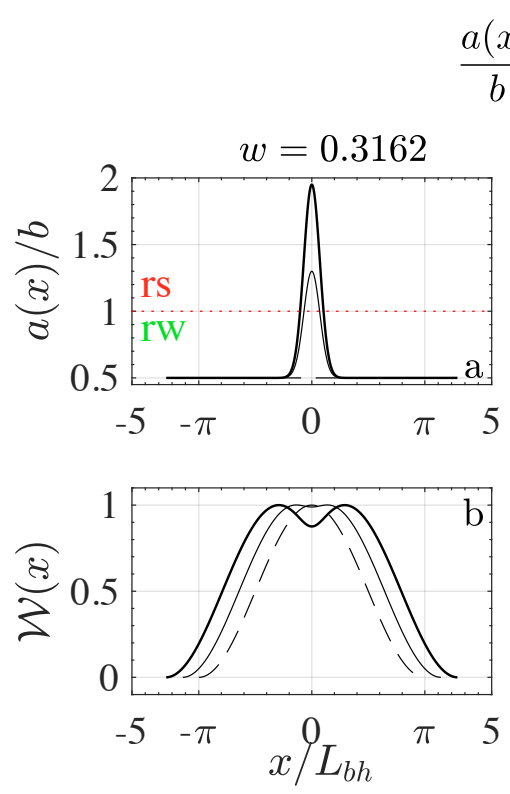

$$
\frac{a(x)}{b}=(a / b)_{o}+\alpha \exp \left(-\frac{\left(x / L_{b h}\right)^{2}}{w^{2}}\right)
$$
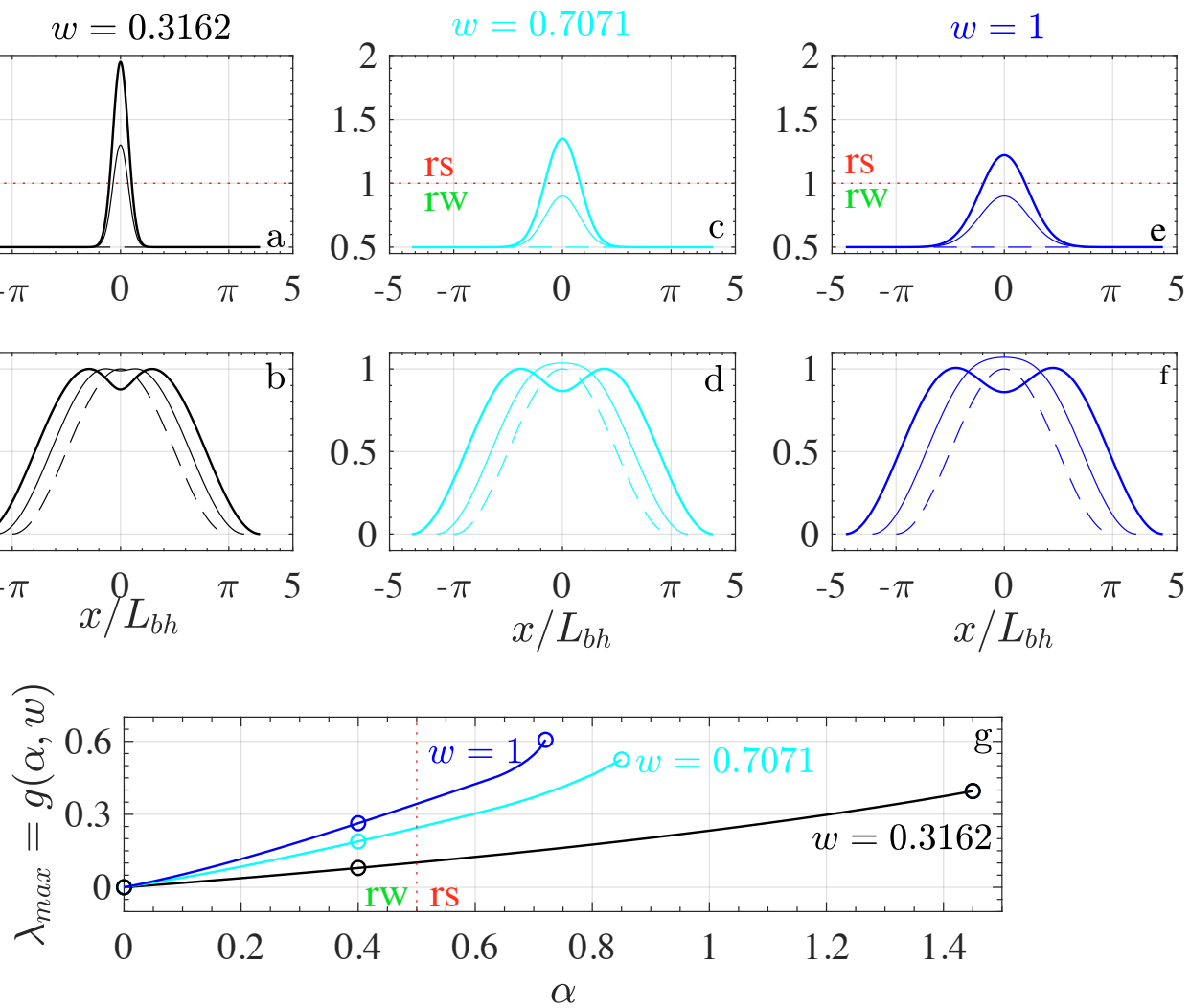

Figure 3. Blow-up solutions when fault friction includes rate-strengthening regions. (a,c,e) We consider heterogeneities of the form $a(x) / b=(a / b)_{o}+\alpha \exp \left(-\frac{x^{2} / L_{b h}^{2}}{w^{2}}\right)$, where $w$ is the width of the Gaussian, $(a / b)_{o}$ is the homogeneous reference, and $(a / b)_{o}+\alpha$ is the peak value. Rate-strengthening and-weakening are abbreviated as rs and rw respectively. (b,d,f) We look for the blow-up solutions for gradually thicker widths $w$. For each width $w$, we find the blow-up solutions for range of peak rate strengthening value $(a / b)_{o}+\alpha$. For each width there exists a critical value of the peak beyond which no blow-up solutions of type (13) are found. (g) The stability of the blow-up solutions as a function of the peak $\alpha$ (in excess of $(a / b)_{o}$ ) is shown for three chosen widths $w$. The circles on the plot correspond to the solutions in (b,d,f).

local type of elastic configurations. Parameter heterogeneities that are solely confined within this range are uninteresting because the discretely distributed solutions are chaotically unstable and an evolving slip rate can not attain any of those solutions. The nucleation of an instability in the near chaotic regime requires very large rate-weakening patch size as indicated in Figure 1.

The two disparate behavior of fixed-points — asymptotically stable and chaotically unstable —are mediated through a transition regime. The transition regime exhibits progressive loss of stability of blow-up solutions through a series of Hopf bifurcations Viesca (2016b). After the third Hopf bifurcation, the slip evolution on homogenous fault becomes chaotic over a large support length (Figure 1). We refer to the regime before the third bifurcation - at most two Hopf bifurcation — as our transition regime. 
In the next section, we focus on spatial variation in the direct-effect parameter

$$
a=a(x)
$$

(equation (1). We consider heterogeneity that includes rate-strengthening regions as well; however, the fault on an average over nucleation scale remains rate-weakening such that the average rate-weakening, $\langle a(x) / b\rangle$, falls within the transition regime, discussed previously. Such heterogeneity, subsequently, play a role in delaying an instability and creating tremor bursts type scenarios.

A monochromatic heterogeneity in parameters may be restrictive to produce a generalized variation. Therefore, we switch to two-parameter Gaussian functions. In particular, we focus on how disparate heterogeneities in the direct-effect parameter might lead to the blow-up solutions whose stability are identical or nearly so.

\subsection{Disparate heterogeneities with similar stability of the blow-up solutions}

Ray \& Viesca $(2017,2019)$ studied monochromatic variations of fault frictional properties and showed that the relevant eigenvalues, $\lambda_{\max }$, are governed by the magnitude and wavelength of the property variation. A question arises: when do two distributions of frictional parameters result in identical maximum eigenvalues? If so, are their stabilities are also identical? Figure (2) aims at this question. The blow-up solutions are solved with heterogeneities governed by two parameters: width $w$ and trough $\alpha$ such that $a(x) / b=0.5+\alpha \exp \left(-\frac{x^{2} / L_{b h}^{2}}{w^{2}}\right)$. We find the maximum eigenvalue to increase with the width and the trough.

Likewise, we again consider $a(x) / b=(a / b)_{o}+\alpha \exp \left(-\frac{x^{2} / L_{b h}^{2}}{w^{2}}\right)$, this time with positive $\alpha$ (Figure 3). We analyze the blow-up solution at the peak of $a(x) / b$. We note that neighbouring regions host stable solutions unlike previous case; however, we focus on a family of solutions of type (13) that are unstable. When the peak value $(a / b)_{o}+\alpha$ is rate strengthening, interestingly, the Newton-Raphson could not converge to a solution like (13), at a prescribed width, for $\alpha$ larger than a critical value. Figure 3 shows that a thinner width allows us to continue the family of solutions to larger peak rate-strengthening values. The corresponding stability curves show that solutions for thicker width are more unstable for any $\alpha$. The plots also show that there are multiple pairs $(w, \alpha)$ that are comparably un-stable.

The above two examples convince us that the eigenvalues $-\lambda_{\max }$ - carries information about the fault frictional heterogeneity and scales with the shape and magnitude. However, owed to their non-uniqueness, a natural question arises whether that dependence could be expressed as a combination of parameters: for example area below $a(x) / b$, product of the peak-value and the width, or average $a / b$. However, we postpone a detailed analysis on how eigenvalues might scale with the parameter combinations for future studies. For the purpose of this work we focus on ways in which a fault's heterogeneity can make solutions (13) unstable.

\section{LOSS OF STABILITY OF THE BLOW-UP SOLUTIONS DELAYS SLIP INSTABILITIES AND MAY CREATE TREMOR BURSTS}

Ray \& Viesca $(2017,2019)$ focused on heterogeneities that yield pairs of blow-up solutions: asymptotically stable and unstable. In those studies unstable solutions were always accompanied by another stable one, such 


$$
\frac{a(x)}{b}=(a / b)_{o}+\alpha \sum_{n} \exp \left(-\frac{(x-n \chi)^{2} / L_{b}^{2}}{2 w^{2}}\right)
$$
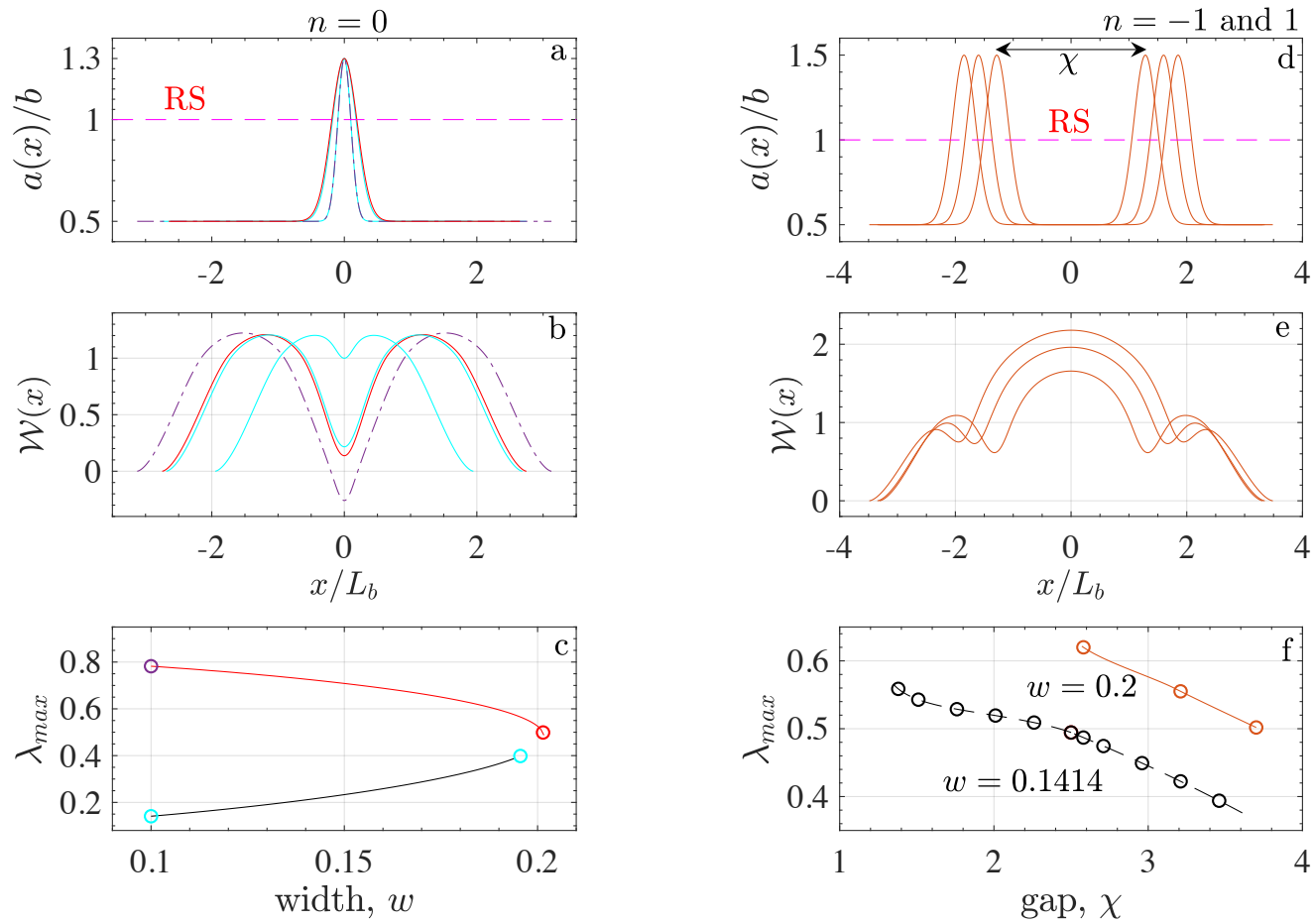

Figure 4. Self-similar solutions of type in the equation (13) that are unstable and choice of parameters that make them so. Nonlocal elastic configuration is considered here. In light of the discussion for next figures, where a series of Gaussianfunctions (20) are used to pose alternating rate-weakening and strengthening patches, we analyze the stability of the blowup solutions at the peak of $\frac{a(x)}{b}=(a / b)_{o}+\alpha \exp \left(-\frac{x^{2} / L_{b}^{2}}{2 w^{2}}\right)$ and between two peaks. We want to identify the width of a peak and the gap between any two peaks that can yield most unstable self-similar solutions. The homogeneous reference is $(a / b)_{o}=0.5$, and $\alpha$ is equal to 0.8 and 1 for (a) and (d) respectively. (a,b,c): We find two families of double-peaked blow-up solutions whose stability curves gradually come closer with increasing width. The red curve in (c) corresponds to a family of double-peaked blow-up solutions with a negative value of trough. The purple and red circles correspond to, respectively, the dashed purple and red blow-up solutions in (b). The black curve corresponds to stability of doublepeaked solutions with positive middle trough. The cyan color circles correspond to cyan colored solutions in (b). The two stability curves about to reach the same point around $w=0.2$. This is also indicated by the closeness of the red and cyan colored solutions in (b). We could not get the Newton-Raphson iteration to converge to a single-peaked or double-peaked blow-up solution $\mathcal{W}(x)$ beyond $w=0.2$ for this case; however, the pattern clearly shows that solutions become more unstable with increasing width. (d,e,f): We look for the gap, $\chi$, between the rate-strengthening peaks that can yield most unstable solutions. Again, we stopped where the iterations could not converge to the desired solutions. The dashed black curve indicates thinner width solutions (not shown) permits shorter gaps between the peaks compared to the thicker width distributions (orange color plots in d).

that an attractive location was always present. We now shift our attention and focus on how the instability development takes place under frictional heterogeneity when only unstable self-similar blow-up solutions are present.

We consider a slip surface within half-spaces and include frictional property variations that include ratestrengthening regions as well. We consider variations of the direct effect coefficient such that its average over a nucleation length, remains rate-weakening, despite including rate-strengthening regions. Specifically we 


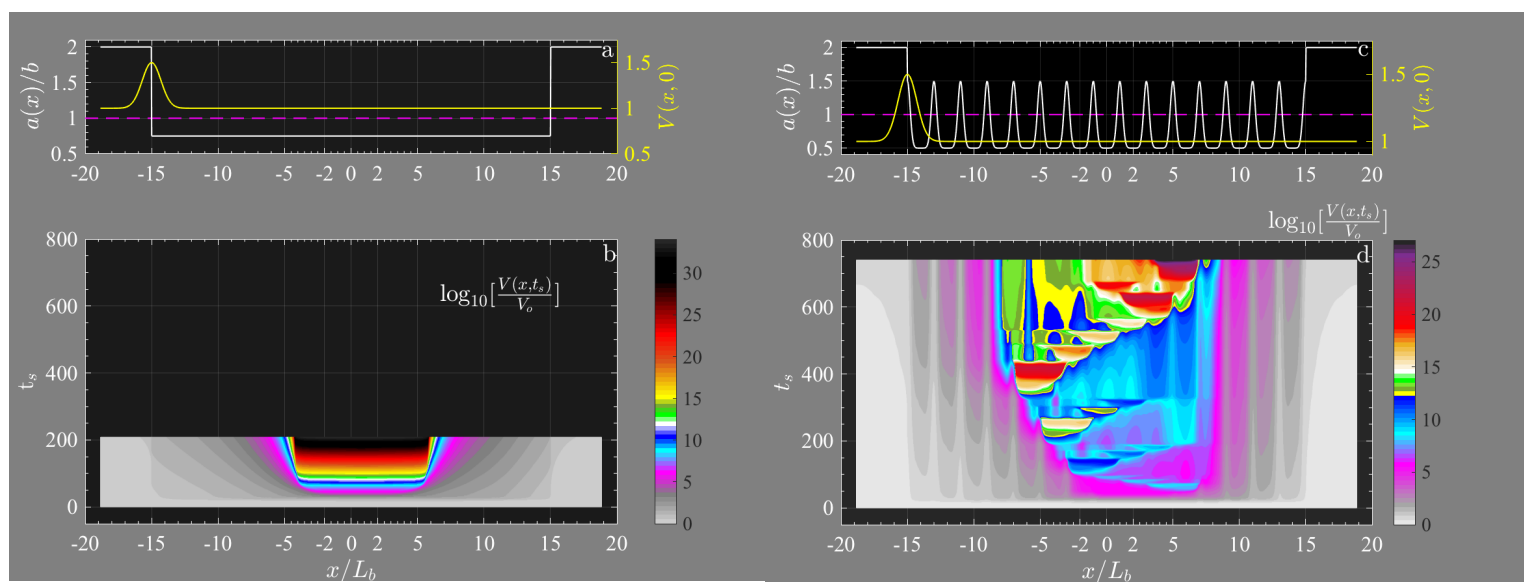

Figure 5. Comparing slip rate evolutions for slip surfaces within two half-spaces underhomogenous with heterogeneities that include rate-strengthening patches. Both the faults $(a, c)$ are identically provoked. $(a, b)$ Homogeneous conditions promote a localized and fast approach to an unbounded slip rate. A fast approach to an unbounded slip rate is also exhibited on purely rate weakening heterogeneous faults (far from the chaotic regime described in section 4.1) at favorable fault locations Ray \& Viesca $(2017,2019)$. Those favorable locations are owed to the existence of asymptotically stable blow-up solutions. (c, d) Using analysis on the unstable blow-up solutions in Figure 4, heterogeneity in (c) includes appropriate rate-strengthening patches such that all the blow-up solutions are unstable. Consequently, the evolving slip oscillates in time as well as in space and span a wide range along the fault compared to the homogeneous case.

consider (series of) shifted shifted and translated Gaussian-functions of the form

$$
\frac{a(x)}{b}=(a / b)_{o}+\alpha \sum_{n} \exp \left(-\frac{(x-n \chi)^{2} / L_{b}^{2}}{2 w^{2}}\right)
$$

where $\alpha$ is such that the peaks, which are separated by $\chi$, are in the rate-strengthening regime. We solve for the blow-up solutions (13) located at the rate-strengthening peaks and between them. We seek the width and gap that can ensure sufficiently unstable solutions: that is large positive $\lambda_{\max }$ values. Figure 4 demonstrates that the blow-up solutions, at the peaks and between them, progressively lose stability as the prescribed heterogeneity leads to a larger degree of rate-strengthening. The results are qualitatively similar for parameter variations of similar nature, for example box car functions; however, our choice for smoother Gaussian functions stems from the need to evaluate spatial derivatives while implementing Newton-Raphson iterative solvers described in section (supplementary information).

The analyses shown in the Figure 4 provides a basis to select appropriate ranges of the thickness and the gap that can yield un-attractive fixed point solutions. Note that an arbitrarily thick $w$ or small $\chi$ could behave just like a homogeneous fault as pointed out by Ray \& Viesca (2019) where an evolving slip could reflect only the average frictional property. Motivated by the results shown in Figure 4, we now numerically integrate system of equations (4) for slip rate evolution (4) using implicit Runge-Kutta time stepping of fourth order. We integrate with non-dimensionalized slip velocity and time. We push the integration limit to a higher dimensionless slip velocities by rescaling time for each time step. We choose a series of Gaussian functions (20) such that their widths, the peak values, and their separation yield blow-up solutions that are un-attractive. We follow Ray \& Viesca (2019) and keep the gap $\chi$ large enough to forbid any homogenization.

Figure 5 compares slip rate evolution under homogeneous conditions with evolution under the hetero- 


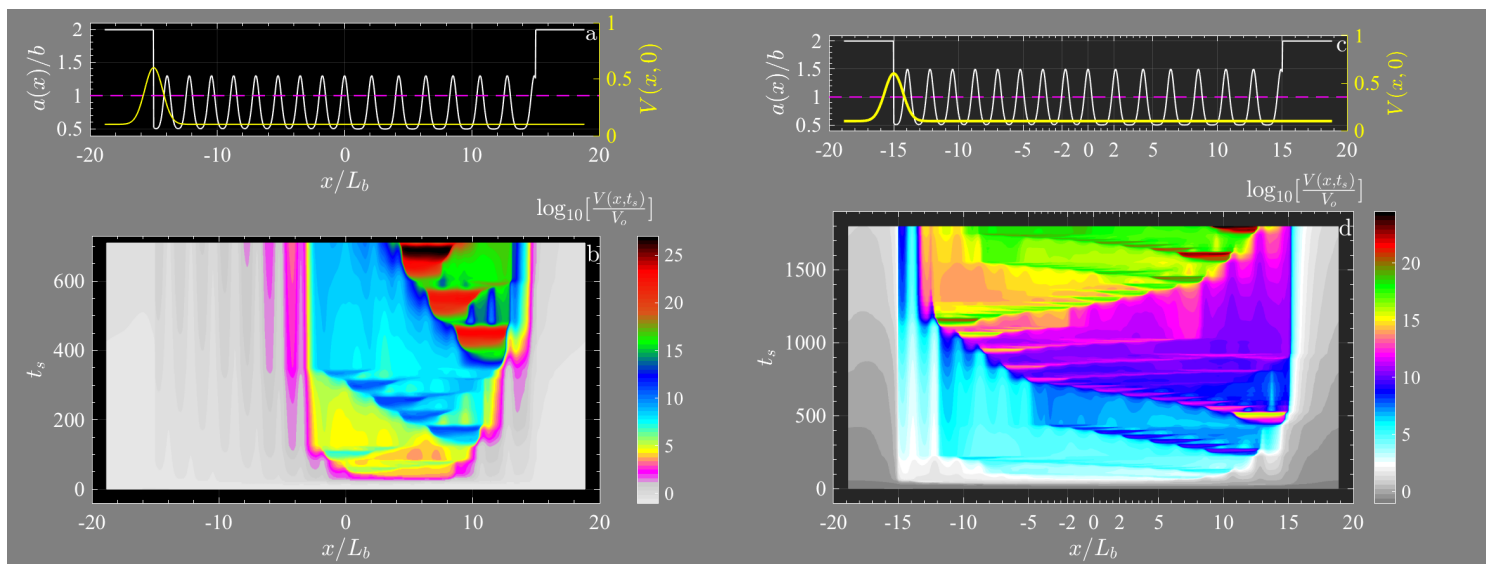

Figure 6. Slip rate evolution for slip surface within half-spaces for two heterogeneities that include rate-strengthening regions. (a, c) Both include rate-strengthening regions with the peak value in (c) larger than that of in (a). The separation between the peaks in the right halves $(x>0)$, in (a) as well as in (c), are slightly higher compared to the left halves.(b)Due to the smaller average value of $a / b$, high slip rates are attained comparatively faster than (d). The larger separation between the peaks in the right half and lower average $a / b$ together enhanced high slip rate activity in the right half. (d) Slightly larger separation between the peaks in the right half $(x>0)$ did not matter in this case owed to the larger average $a / b$. Consequently, the spatio-temporal oscillation of slip spanned a wide range along the fault.

geneous conditions just described. The slip evolves in strikingly different manner for the two cases. Both the faults are at steady-state when a small initial velocity perturbation is implemented at one end. An instability nucleates within a short time on the homogeneous fault. This is a consequence of an existing attractive blow-up solution, to which the evolving slip-rate converges. On the contrary, all the blow-up solutions for the heterogenous case are un-attractive owed to one or more strongly unstable modes (like in Figure 4). Such modes are easily provoked due to nonuniform parameter distributions, which, in turn, lead to apparent non-convergence of the evolving slip-rate Figure $5 \mathrm{~d}$. Further, unlike the homogeneous case, the faults' heterogeneity and non-existent attractive solutions promote interactions within large regions on the fault.

Figure 6 demonstrates two heterogenous situations with 6a more rate-weakening compared the 6c. All the blow-up solutions in both the cases are unstable; however, Figure 6(c,d) is associated with stronger unstable modes due to the large peak-values in the distribution of $a(x) / b$ (also see Figure 4). High slip rates are attained faster in Figure 6(a,b). Like the previous case, heterogeneity and absent attractive fixed point solutions promote increased quasi-static interactions within large regions on the fault.

\section{SUMMARY}

We considered non-monochromatic variations of frictional properties and focused on scenarios that can lead to unstable self-similar blow-up solutions. We found that faults with frictional heterogeneities that include considerable rate-strengthening region can cause blow-up solutions to lose stability. As a result, owed to the lack of any attractor, an evolving slip rate cannot localize to a specific location on the fault, and consequently, assumes a near-chaotic evolution. Figure 7 demonstrates an instance of near-chaotic slip rate evolution and 


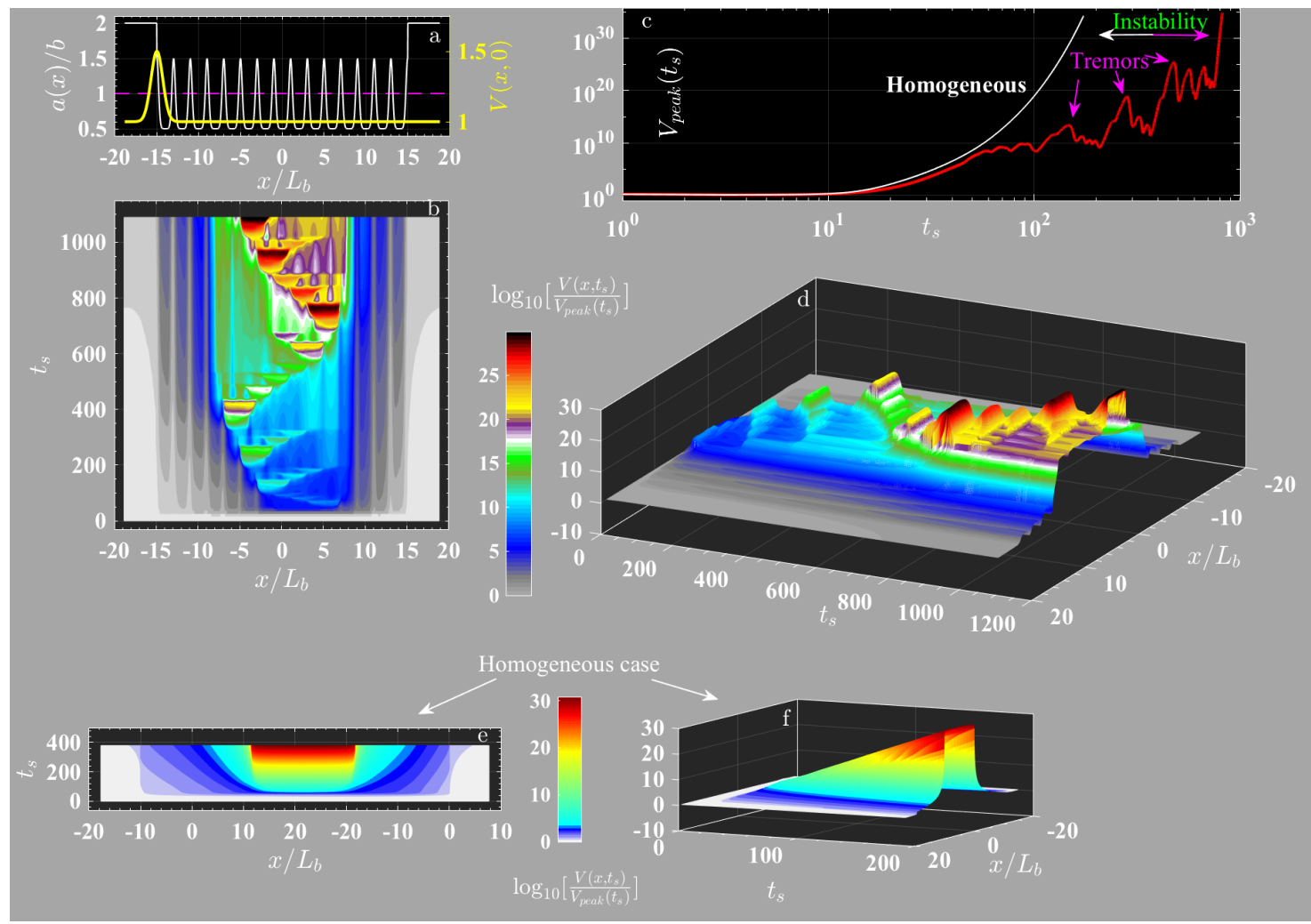

Figure 7. We consider slip evolution for full-space elastic configuration under frictional heterogeneity that includes ratestrengthening regions (a,b,c,d). The results are compared against the homogenous case (e, f). The plots (d) and (f), respectively, are quantitatively same as (b) and (e). The plots in (c) compare the peak values of slip velocity for the two cases: In homogenous case, the slip rate diverges monotonically, however, the peak slip rate for the heterogenous case shows unpredictable changes.Unlike the homogeneous case (e, f), the apparent near-chaotic slip evolution in $(b, d)$ is purely owed to the absence of any stable blow-up solution.

compares with slip evolution under a homogenous case. Heterogeneities that promote unstable blow-up solutions can cause distributed tremors on the fault and can delay earthquake nucleating slip instabilities.

\section{ACKNOWLEDGEMENTS}

This manuscript, after an update, will be submitted in Geophysical Journal International. I thank Allan Rubin for discussion. I also thank Robert C. Viesca for his suggestions and comments that improved the overall presentation. 


\section{References}

Dieterich, J. H., 1978a. Time-dependent friction and the mechanics of stick-slip, Pure and Applied Geophysics, 116(4), 790-806.

Dieterich, J. H., 1978b. Time-dependent friction and the mechanics of stick-slip, Pure and Applied Geophysics, 116(4-5), 790-806.

Dieterich, J. H., 1979. Modeling of rock friction: 1. experimental results and constitutive equations, Journal of Geophysical Research: Solid Earth, 84(B5), 2161-2168.

Erdogan, F., Gupta, G. D., \& Cook, T. S., 1973. Numerical solution of singular integral equations, pp. 368-425, Springer Netherlands, Dordrecht.

Garagash, D. I. \& Germanovich, L. N., 2012. Nucleation and arrest of dynamic slip on a pressurized fault, Journal of Geophysical Research: Solid Earth, 117(B10).

Kato, N. \& Tullis, T. E., 2001. A composite rate- and state-dependent law for rock friction, Geophysical Research Letters, 28(6), 1103-1106.

Nagata, K., Nakatani, M., \& Yoshida, S., 2012. A revised rate-and state-dependent friction law obtained by constraining constitutive and evolution laws separately with laboratory data, Journal of Geophysical Research: Solid Earth, 117(B2).

Ray, S. \& Viesca, R. C., 2017. Earthquake nucleation on faults with heterogeneous frictional properties, normal stress, Journal of Geophysical Research, 122(10), 8214-8240.

Ray, S. \& Viesca, R. C., 2019. Homogenization of fault frictional properties, Geophysical Journal International. Rice, J. R. \& Ruina, A. L., 1983. Stability of steady frictional slipping, J. Appl. Mech., 50, 343-349.

Rubin, A. M. \& Ampuero, J.-P., 2005. Earthquake nucleation on (aging) rate and state faults, Journal of Geophysical Research: Solid Earth, 110, B11312.

Ruina, A., 1983. Slip instability and state variable friction laws, Journal of Geophysical Research, 88(B12), 10359-10370.

Tullis, T. E. \& Weeks, J. D., 1986. Constitutive behavior and stability of frictional sliding of granite, Pure and Applied Geophysics, 124(3), 383-414.

Uenishi, K. \& Rice, J. R., 2003. Universal nucleation length for slip-weakening rupture instability under nonuniform fault loading, Journal of Geophysical Research: Solid Earth, 108(B1).

Viesca, R. C., 2016a. Self-similar slip instability on interfaces with rate-and state-dependent friction, in Proc. R. Soc. A, vol. 472, p. 20160254, The Royal Society.

Viesca, R. C., 2016b. Stable and unstable development of an interfacial sliding instability, Physical Review E, 93(6), 060202.

Viesca, R. C. \& Garagash, D. I., 2018. Numerical methods for coupled fracture problems, Journal of the Mechanics and Physics of Solids, 113, 13-34.

Weber, C. A., Rycroft, C. H., \& Mahadevan, L., 2018. Differential activity-driven instabilities in biphasic active matter, Physical review letters, 120(24), 248003. 


\section{Supplementary materials for "Frictional heterogeneities can promote disordered slip evolution on faults"}

\section{Sohom Ray}

Civil and Environmental Engineering, Tufts University, Medford, MA 02155, USA

Sohom.Ray@tufts.edu

\section{September 2019}

\section{FAULT'S FRICTIONAL STRENGTH: SLIP RATE AND STATE DEPENDENT FRICTION}

We assume that the strength of the fault is purely frictional, given by,

$$
\tau_{s}(x, t)=\sigma(x, t) f(x, t)
$$

where $\sigma$ is the fault-normal stress and $f$ is the friction coefficient. In the case of a fluid-saturated fault zone, $\sigma$ is the effective fault-normal stress, given by $\sigma(x, t)=\sigma_{n}(x, t)-p(x, t)$, where $\sigma_{n}$ is the total normal stress and $p$ is the pore fluid pressure on the fault surface.

We consider a rate- and state-dependent constitutive formulation of frictional strength (e.g., Dieterich, 1978, 1979; Rice \& Ruina, 1983). In this framework, the coefficient of friction $f$ at a position on the fault is a function of the instantaneous slip-rate $V=\partial \delta / \partial t$, a state variable $\theta$, and material parameters $a$ and $b$ at that position, and is expressed as

$$
f(x, t)=f_{o}+a \ln \left[\frac{V(x, t)}{V_{o}}\right]+b \ln \left[\frac{\theta(x, t)}{D_{c} / V_{o}}\right]
$$

where $f_{o}$ is the reference coefficient of friction at steady-state sliding velocity $V_{o}$.

The dimensionless parameters, $a$ and $b$, are the properties of the contacting surfaces. The parameter $a$ moderates the magnitude of the immediate change of friction in response to a sudden jump in steady state sliding velocity, and is referred to as the direct effect coefficient. Following the instantaneous jump, the friction coefficient subsequently relaxes, over a sliding distance of $D_{c}$, to a renewed steady state value. The parameter $b$ moderates the magnitude of the relaxation effect and referred to as an evolution effect coefficient.

The state variable $\theta$ - has a dimension of time — contains information on the history or changes in sliding rate. At a particular sliding rate the steady-state value of the state variable is given by

$$
\theta=\theta_{s s}=\frac{D_{c}}{V}
$$

The quantity $D_{c} / V$ can also interpreted as a time scale that preserves the memory of the sliding conditions. We extend that notion and consider the possibility of a general delay time scale: $t_{D}$. Any memory or change in sliding condition permeates into the frictional strength through the evolution of $\theta$, given by,

$$
t_{D} \frac{\partial \theta}{\partial t}+P(V, \theta) \theta=Q(V, \theta)
$$



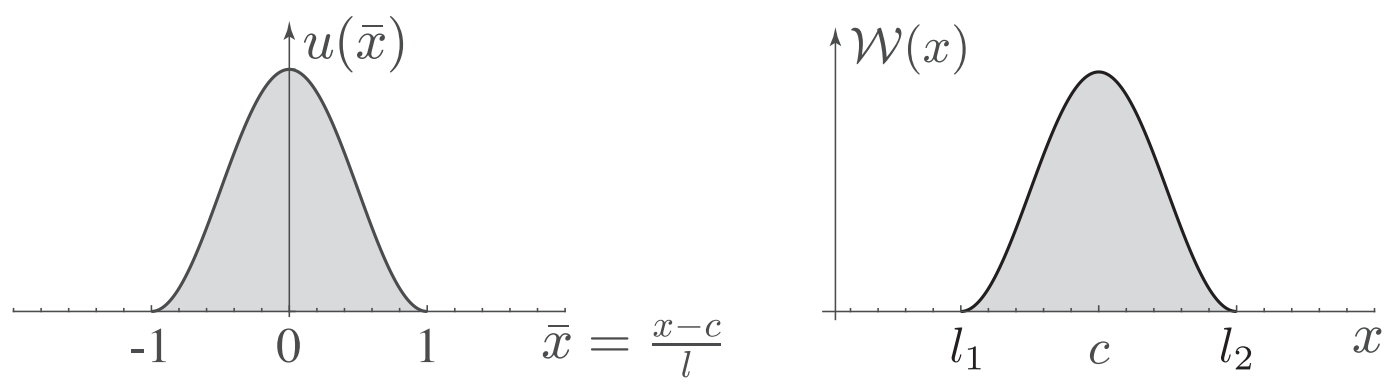

Figure 1. We recast the problems (7) and (15) in a new coordinate using the variable transformation $x=l \bar{x}+c$ where $\bar{x} \equiv[-1,1], l=\frac{1}{2}\left(l_{2}-l_{1}\right)$, and $c=\frac{1}{2}\left(l_{1}+l_{2}\right)$, and look for the solutions of the type $\mathcal{W}(x)=u\left(\frac{x-c}{l}\right)=u(\bar{x})$ and $\mathcal{P}(x)=p\left(\frac{x-c}{l}\right)=p(\bar{x})$.

Based on the laboratory rock friction experiments, few empirical rules for evolution of $\theta$ with time are proposed (e.g., Dieterich, 1978, 1979; Ruina, 1983; Tullis \& Weeks, 1986; Kato \& Tullis, 2001; Nagata et al., 2012). They share one common attribute that the time scale of state evolution is $t_{D}=D_{c} / V$. The terms $P(V, \theta)$ and $Q(V, \theta)$ for the commonly discussed evolution laws are shown below

$$
\begin{aligned}
\text { Aging: } t_{D} & =\frac{D_{c}}{V}, Q(V, \theta)=\frac{D_{c}}{V}, \quad P(V, \theta)=1 \\
\text { Slip: } t_{D} & =\frac{D_{c}}{V}, Q(V, \theta)=0, \quad P(V, \theta)=\ln \left(\frac{V \theta}{D_{c}}\right) \\
\text { Kato and Tullis: } t_{D} & =\frac{D_{c}}{V}, Q(V, \theta)=\frac{D_{c}}{V} e^{-\frac{V}{V_{c}}}, P(V, \theta)=\ln \left(\frac{V \theta}{D_{c}}\right) \\
\text { Nagata: } t_{D} & =\frac{D_{c}}{V}, Q(V, \theta)=(1+n) \frac{D_{c}}{V}, P(V, \theta)=\frac{n}{1+n}\left[1+\frac{a}{b} \frac{D_{c}}{V^{2}} \frac{\partial V}{\partial t}\right]
\end{aligned}
$$

The last two state evolution rules, Kato-Tullis and Nagata, use additional parameters $V_{c}$ and $n$ respectively and we include them for the sake of completeness.

Here we considered the aging-law for state evolution (Dieterich, 1979), which we re-write as,

$$
\frac{D_{c}}{V} \frac{\partial \theta}{\partial t}+\theta=\frac{D_{c}}{V}
$$

\section{METHODS TO SOLVE FOR THE BLOW-UP SOLUTIONS FOR SLIP RATE}

We now move to solve for the self-similar solutions of slip rate (eq. (13), main document), which are also referred to as blow-up or fixed point solutions. The numerical methods to solve for the self-similar blow-up solutions, discussed below, consider heterogeneity only in the direct effect parameter $a=a(x)$. The methods, however, also apply to the cases with variable evolution effect parameter $b=b(x)$, normal stress $\sigma=\sigma(x)$, and slip scale $D_{c}=D_{c}(x)$.

\subsection{Thin SLAB ELASTIC CONFIGURATION}

We now proceed to discuss the method to solve for the blow-up solutions: the spatial distribution $\mathcal{W}(x)$ and its extent on the fault. For thin slab elastic configurations, using $x / L_{b h} \rightarrow x$, the problem (eq. (12), main document) reduces to solve an ordinary differential equation whose boundaries are also unknowns of the 
problem, given by,

$$
\begin{aligned}
& \frac{d^{2} \mathcal{W}(x)}{d x^{2}}+\mathcal{W}(x) \mathcal{P}(x)-\frac{a(x)}{b}=0 \\
& \mathcal{W}\left(l_{1}\right)=0, \mathcal{W}\left(l_{2}\right)=0 \\
& \mathcal{W}^{\prime}\left(l_{1}\right)=0, \mathcal{W}^{\prime}\left(l_{2}\right)=0
\end{aligned}
$$

wherein the distribution $\mathcal{W}(x)$, and the boundaries $l_{1}$ and $l_{2}$ are to be determined. We note that the function $\mathcal{W}(x) \mathcal{P}(x)$ is equal to either 1 or $\mathcal{W}(x)$ depending upon whether $\mathcal{W}(x)$ is greater or lesser than unity. That is,

$$
\mathcal{P}(x)= \begin{cases}1, & \mathcal{W}(x) \leq 1 \\ \frac{1}{\mathcal{W}(x)}, & \mathcal{W}(x)>1\end{cases}
$$

In case of homogeneous properties, the problem simplifies to a symmetric distribution $\mathcal{W}(x)$ and its support with half-length $l=-l_{2}=l_{1}$. We note that multiple pairs $(\mathcal{W}(x), l)$ exist that satisfy the above equation. However, our interest lies on the blow-up solutions (eq. (13), main document) with minimum support length: larger support length solutions are unattainable. Likewise, under heterogenous conditions, multiple triplets $\left(\mathcal{W}(x), l_{1}, l_{2}\right)$ satisfy the above equations and we look for the solutions where $\mathcal{W}(x)$ remains nonzero within $l_{1}$ and $l_{2}$.

To find them, the problem (eq. (12), main document) is discretized into a set of non-linear algebraic equations and the solution found by iterative Newton-Raphson root finding. The convergence to the desired solution under nonuniform $a(x) / b$ is complicated owed to broken translational invariance: that is, multiple solutions for $\mathcal{W}(x)$ exist at different locations in space.

To ensure convergence of the Newton-Raphson iterations, we recast the problem (7) in a translating coordinates, that is, using the variable transformation $x=l \bar{x}+c$ where $\bar{x} \equiv[-1,1], l=\frac{1}{2}\left(l_{2}-l_{1}\right)$, and $c=\frac{1}{2}\left(l_{1}+l_{2}\right)$, and look for the solutions of the form

$$
\begin{aligned}
& \mathcal{W}(x)=u\left(\frac{x-c}{l}\right)=u(\bar{x}) \\
& \mathcal{P}(x)=p\left(\frac{x-c}{l}\right)=p(\bar{x})
\end{aligned}
$$

The problem reduces to the following system: a differential equation under Dirichlet boundary conditions, and two conditions on $u(\bar{x})$ at the boundaries which prevents a singularity in the stress-rate at the boundaries.

$$
\begin{aligned}
U[u(\bar{x}), l, c] \equiv & \frac{1}{l^{2}} \frac{d^{2} u(\bar{x})}{d \bar{x}^{2}}+u(\bar{x}) p(\bar{x})-\frac{a(l \bar{x}+c)}{b}=0, \\
& \text { with } u(-1)=0 \text { and } u(1)=0 \\
L[u(\bar{x}), l, c] \equiv & \left.\frac{d u(\bar{x})}{d \bar{x}}\right|_{\bar{x}=-1}=0, \\
C[u(\bar{x}), l, c] \equiv & \left.\frac{d u(\bar{x})}{d \bar{x}}\right|_{\bar{x}=+1}=0 .
\end{aligned}
$$


where we note that the function $p(\bar{x})$, is given by,

$$
p(\bar{x})= \begin{cases}1, & u(\bar{x}) \leq 1 \\ \frac{1}{u(\bar{x})}, & u(\bar{x})>1\end{cases}
$$

The spatial discretizations, based on the finite difference schemes, and the Newton-Raphson iterations to solve for the nonlinear system (10) are are discussed next.

\subsection{NEWTON RAPHSON ITERATION FOR THIN-SLAB ELASTIC CONFIGURATION}

The domain is discretized uniformly: $\bar{x}_{i}=-1+i \Delta \bar{x}$, where $i=1, \ldots, N$. The central-difference scheme is utilized for second-order derivative $\left(D_{i j}\right)$, forward and backward difference schemes with second-order accuracy are used, respectively, for the first order derivatives at the left and right boundaries. Thus, the discretized form of the above system of equations, is given by,

$$
\begin{aligned}
U_{i}\left[u_{i}, l, c\right] & \equiv \frac{1}{l^{2}} D_{i j} u_{j}+u_{i} p_{i}-\frac{a_{i}}{b}=0, \quad u_{1}=0=u_{N}, \\
L\left[u_{i}, l, c\right] & \equiv 2 u_{2}-0.5 u_{3}=0, \\
C\left[u_{i}, l, c\right] & \equiv-2 u_{N-1}+0.5 u_{N-2}=0 .
\end{aligned}
$$

where $a_{i}=a\left(l \bar{x}_{i}+c\right)$. Note that $u_{1}=0$ and $u_{N}=0$ are utilized in the last two equations. We note that where we note that the function $u_{i} p_{i}$ is equal to either 1 or $u_{i}$ depending upon whether $u_{i}$ is greater or lesser than unity. That is,

$$
p_{i}=\left\{\begin{array}{l}
1, u_{i} \leq 1 \\
\frac{1}{u_{i}}, u_{i}>1
\end{array}\right.
$$

We arrive to solutions of the non-linear system (12) by Newton-Raphson iteration. Following an initial guess for the distribution $u_{i}$, half-length $l$ and the center $c$, the updated $u_{i}, l$ and $c$ are obtained iteratively using Newton-Raphson iterations.

$$
\left[\begin{array}{c}
u_{i} \\
l \\
c
\end{array}\right]_{n+1}=\left[\begin{array}{c}
u_{i} \\
l \\
c
\end{array}\right]_{n}-g G_{n}^{-1}\left[\begin{array}{c}
U_{i} \\
L \\
C
\end{array}\right]_{n}
$$

where $g<1$ allows smaller increments in the iterations, and, as a result, a slower (but often ensured) convergence to the fixed-point solution of the iteration. The gradient operator $G$ is given by,

$$
\begin{aligned}
G_{n} & =\left[\begin{array}{lll}
\partial_{u} U & \partial_{l} U & \partial_{c} U \\
\partial_{u} L & \partial_{l} L & \partial_{c} L \\
\partial_{u} C & \partial_{l} C & \partial_{c} C
\end{array}\right]_{n} \\
& =\left[\begin{array}{ccc}
{\left[\frac{1}{l^{2}} D_{i j}+I_{i j}\right]_{N \times N}} & {\left[-\frac{2}{l^{3}} D_{i j} u_{j}-a_{i}^{\prime} . x_{i}\right]_{N \times 1}} & {\left[-a_{i}^{\prime}\right]_{N \times 1}} \\
{[0,2,-0.5,0, \ldots, 0]_{1 \times N}} & 0 & 0 \\
{[0, \ldots, 0,0.5,-2,0]_{1 \times N}} & 0 & 0
\end{array}\right]_{n}
\end{aligned}
$$


where the differentiation with respect to $u$ are to be interpreted in Frechet derivative sense. $I_{i j}$ is an $N \times N$ diagonal matrix with diagonal entries equal to unity when $p_{i}=1$ and equal to zero when $u_{i} p_{i}=1$. Once the distributions $u(\bar{x}), l$ and $c$ are obtained the blow-up solution $\mathcal{W}(x)$, and its extent, $l_{1}$ and $l_{2}$, are obtained by using equations (9).

\subsection{ELASTIC HALF-SPACE TYPE CONFIGURATION}

In case of slip surface within two elastic half-spaces the problem (eq. (12), main document) reduces to an integro-differential equation with distribution $\left.\mathcal{W}(x), l_{1}\right]$ and $l_{2}$, are unknowns. We note that the coordinate $x$ in this case is expressed in the units of $L_{b}$ (and not $L_{b h}$ ). The system of equations to solve for is given by

$$
\begin{aligned}
& \frac{1}{2 \pi} \int_{l_{1}}^{l_{2}} \frac{d \mathcal{W}(y) / d y}{y-x} d y+\mathcal{W}(x) \mathcal{P}(x)-\frac{a(x)}{b}=0 \\
& \mathcal{W}\left(l_{1}\right)=0, \mathcal{W}\left(l_{2}\right)=0, \\
& \mathcal{W}^{\prime}\left(l_{1}\right)=0, \mathcal{W}^{\prime}\left(l_{2}\right)=0
\end{aligned}
$$

where the function $\mathcal{P}(x)$ is given by the equation (8) with $x$ to be interpreted in the units of the lengthscale $L_{b}$. We again look for the blow-up solutions (eq. (13), main document), triplets $\left(\mathcal{W}(x), l_{1}, l_{2}\right)$, that satisfy the above equations such that $\mathcal{W}(x)$ remains nonzero within $l_{1}$ and $l_{2}$. We again switch to translating coordinate system: $x=l \bar{x}+c$ where $\bar{x} \equiv[-1,1], l=\frac{1}{2}\left(l_{2}-l_{1}\right)$, and $c=\frac{1}{2}\left(l_{1}+l_{2}\right)$, and find the solutions (9). In the new coordinates, the above problem reduces to the following system of equations:

$$
\begin{aligned}
& U[u(\bar{x}), l, c] \equiv \frac{1}{2 \pi l} \int_{-1}^{1} \frac{d u(\bar{y}) / d \bar{y}}{\bar{y}-\bar{x}} d \bar{y}+u(\bar{x}) p(\bar{x})-\frac{a(l \bar{x}+c)}{b}=0, \\
& \text { with } u(-1)=0 \text { and } u(1)=0 \\
& \left.L[u(\bar{x}), l, c] \equiv \frac{d u(\bar{x})}{d \bar{x}}\right|_{\bar{x}=-1}=0 \\
& \left.C[u(\bar{x}), l, c] \equiv \frac{d u(\bar{x})}{d \bar{x}}\right|_{\bar{x}=+1}=0 .
\end{aligned}
$$

where we note that the function $u(\bar{x}) p(\bar{x})$ is equal to either 1 or $u(\bar{x})$ depending upon whether $u(\bar{x})$ is greater or lesser than unity (as shown in the equation (11)). A Chebyshev discretization, evaluation of the quadrature, and the Newton-Raphson implementation are discussed next.

\subsection{Hilbert TRANSForm, Chebyshev POlynomials, GaUSS-ChEbYSHEV QUADRATURE}

We now construct a numerical method to solve the system of equations (16) for the blow-up solution and its support. We implicitly use the equilibrium conditions $(16 \mathrm{~b}, \mathrm{c})$ to express the gradient inside the integral as

$$
\frac{d u}{d \bar{y}}=\sqrt{1-\bar{y}^{2}} F(\bar{y})
$$

where the choice of $\sqrt{1-\bar{y}^{2}}$ instead of any other function that yields zero gradient at the ends is motivated from slip's scaling near the crack tips of a non-singular crack (e.g., Uenishi \& Rice, 2003; Viesca \& Garagash, 
2018). Therefore, $u(\bar{x})$ is given by

$$
u(\bar{x})=u(-1)+\int_{-1}^{\bar{x}} \sqrt{1-\bar{y}^{2}} F(\bar{y}) d \bar{y}
$$

Using a Heaviside step function, given by,

$$
\mathcal{H}(\bar{y}-\bar{x})=\left\{\begin{array}{l}
0, \bar{y}<\bar{x} \\
1, \bar{y}>\bar{x}
\end{array}\right.
$$

and the condition $u(-1)=0$, the above expression can be re-written as,

$$
u(\bar{x})=\int_{-1}^{1} \mathcal{H}(\bar{x}-\bar{y}) \sqrt{1-\bar{y}^{2}} F(\bar{y}) d \bar{y}
$$

where we have used the following property of a Heaviside function:

$$
\mathcal{H}(\bar{x}-\bar{y})=1-\mathcal{H}(\bar{y}-\bar{x}) .
$$

At $\bar{x}=1$ the function $\mathcal{H}(1-\bar{y})=1$ uniformly for all $\bar{y}$ and $u(1)=0$. Using this, and recalling that we have utilized equations (16b,c) and $u(-1)=0$ implicitly, the system of equations (16) simplifies to the following

$$
\begin{aligned}
& N[F(\bar{y}), l, c] \equiv \frac{1}{2 \pi l} \int_{-1}^{1} \frac{\sqrt{1-\bar{y}^{2}} F(\bar{y})}{\bar{y}-\bar{x}} d \bar{y}+p(\bar{x}) \int_{-1}^{1} \mathcal{H}(\bar{x}-\bar{y}) \sqrt{1-\bar{y}^{2}} F(\bar{y}) d \bar{y}-\frac{a(l \bar{x}+c)}{b}=0, \\
& R[F(\bar{y}), l, c] \equiv \int_{-1}^{1} \sqrt{1-\bar{y}^{2}} F(\bar{y}) d \bar{y}=0
\end{aligned}
$$

where we note that the function $p(\bar{x})$ is equal to either 1 or $1 / u(\bar{x})$ depending upon whether $u(\bar{x})$ is less or greater than unity (11). We follow Erdogan et al. (1973) and Viesca \& Garagash (2018) and references therein, to numerically evaluate the integrals (21). We recall the (trignometric) definitions of Chebyshev polynomials of first and second kind defined as, respectively,

$$
\begin{aligned}
T_{n}(\bar{y}) & =\cos \left[n \cos ^{-1}(\bar{y})\right] \\
\text { and } U_{n-1}(\bar{y}) & =\frac{\sin \left[n \cos ^{-1}(\bar{y})\right]}{\sqrt{1-\bar{y}^{2}}} .
\end{aligned}
$$

where $|\bar{y}| \leq 1$ and $n$ is the order of the Chebyshev polynomials. The Hilbert transform of the weighted Chebyshev polynomials have conjugate relations, given by,

$$
\begin{aligned}
& \frac{1}{\pi} \int_{-1}^{1} \frac{T_{j}(\bar{y})\left(1-\bar{y}^{2}\right)^{-1 / 2}}{\bar{y}-\bar{x}} d \bar{y}=U_{j-1}(\bar{x}) \\
& \frac{1}{\pi} \int_{-1}^{1} \frac{U_{j}(\bar{y})\left(1-\bar{y}^{2}\right)^{1 / 2}}{\bar{y}-\bar{x}} d \bar{y}=-T_{j+1}(\bar{x})
\end{aligned}
$$

Following Erdogan et al. (1973) we discretize the independent variable as

$$
\bar{y}_{k}=\cos \left(\pi \frac{k}{N+1}\right)
$$


and express the integral on the left of (22b) in Gauss-Chebyshev summation form which is given by

$$
\sum_{k=1}^{N} \frac{\left(1-\bar{y}_{k}^{2}\right) U_{j}\left(\bar{y}_{k}\right)}{(N+1)\left(\bar{y}_{k}-x_{r}\right)} \approx-T_{j+1}\left(x_{r}\right)
$$

We recall from section that we seek to numerically approximate the following integrals,

$$
\int_{-1}^{1} \sqrt{1-\bar{y}^{2}} F(\bar{y}) d \bar{y} \text { and } \quad \int_{-1}^{1} \frac{\sqrt{1-\bar{y}^{2}} F(\bar{y})}{\bar{y}-\bar{x}} d \bar{y}
$$

We discretize the independent variables as

$$
\bar{y}_{k}=\cos \left(\pi \frac{k}{N+1}\right) \quad \text { and } \quad \bar{x}_{r}=\cos \left(\pi \frac{r-1 / 2}{N+1}\right)
$$

where $k=1,2, \ldots, N$ and $r=1,2, \ldots, N+1$. Recognizing that $\sqrt{1-\bar{y}^{2}}$ is the weight used to express the orthogonality relations for Chebyshev polynomials of second kind, $U_{j}(\bar{y})$, we expand $F(\bar{y})$ on the basis of Chebyshev polynomials of the second kind $U_{j}(\bar{y})$ as

$$
F(\bar{y}) \approx \sum_{j=0}^{p} \alpha_{j} U_{j}(\bar{y})
$$

where $U_{j}(\bar{y})$ is given by,

$$
U_{j}(\bar{y})=\frac{\sin \left((j+1)\left(\cos ^{-1} \bar{y}\right)\right)}{\sin \left(\cos ^{-1} \bar{y}\right)}
$$

Following Erdogan et al. (1973) we can approximate the integrals (23) using following Gauss-Chebyshev quadratures

$$
\begin{aligned}
\int_{-1}^{1} \frac{\sqrt{1-\bar{y}^{2}} F(\bar{y})}{\bar{y}-\bar{x}} d \bar{y} & \approx \sum_{j=0}^{N} \alpha_{j} \int_{-1}^{1} \frac{\sqrt{1-\bar{y}^{2}} U_{j}(\bar{y})}{\bar{y}-\bar{x}} d \bar{y} \\
& =-\sum_{j=0}^{N} \alpha_{j} T_{j+1}(\bar{x})
\end{aligned}
$$

where we have used (22b) property of the Chebyshev polynomials. Now using the previous relations we get

$$
\begin{aligned}
\int_{-1}^{1} \frac{\sqrt{1-\bar{y}^{2}} F(\bar{y})}{\bar{y}-\bar{x}} d \bar{y} & =\sum_{j=0}^{p} \sum_{k=1}^{N} \frac{\left(1-\bar{y}_{k}^{2}\right) U_{j}\left(\bar{y}_{k}\right)}{(N+1)\left(\bar{y}_{k}-x_{r}\right)} \\
& \approx \sum_{k=1}^{N} \frac{\left(1-\bar{y}_{k}^{2}\right) F\left(\bar{y}_{k}\right)}{(N+1)\left(\bar{y}_{k}-x_{r}\right)}
\end{aligned}
$$

and

$$
\int_{-1}^{1} \sqrt{1-\bar{y}^{2}} F(\bar{y}) d \bar{y} \approx \sum_{k=1}^{N} \frac{\left(1-\bar{y}_{k}^{2}\right) F\left(\bar{y}_{k}\right)}{N+1}
$$

We use the matrix $\mathcal{T}_{r k}$ for the Hilbert transform summation (26) and a diagonal matrix $\mathcal{C}_{v k}$ for the summation in the Equation (28)

$$
\begin{aligned}
T_{r k} & =\frac{1-\bar{y}_{k}^{2}}{(N+1)\left(\bar{y}_{k}-\bar{x}_{r}\right)} \\
C_{v k} & =\left(1-\bar{y}_{k}^{2}\right) \delta_{v k}^{D}
\end{aligned}
$$

where $x_{r}$ and $y_{k}$ are given by equation (24) and $\delta_{v k}^{D}$ is the Kronecker delta which is unity when $v=k$ and zero 
when $v \neq k$. Further, we express $\mathcal{H}(\bar{x}-\bar{y})$, inside the second integral in the equation (21) using the matrix $\mathcal{H}_{r v}$ (with a minor abuse of notation), given by,

$$
\mathcal{H}\left(\bar{x}_{r}-\bar{y}_{v}\right)=\mathcal{H}_{r v}=\left\{\begin{array}{l}
1, \bar{y}_{v}<\bar{x}_{r} \\
0, \bar{y}_{v}>\bar{x}_{r}
\end{array}\right.
$$

where $x_{r}$ and $y_{v}$ are given by the Equation (24) with subscript $k$ replaced by subscript $v$.

We rewrite the discretized version of (21)

$$
\begin{aligned}
N_{r}\left[F\left(\bar{y}_{k}\right), l, c\right] & \equiv \frac{1}{2 l} \mathcal{T}_{r k} F_{k}+\frac{p_{r}}{n+1}\left(\mathcal{H}_{r v} \mathcal{C}_{v k}\right) F_{k}-a_{r}=0 \\
R\left[F\left(\bar{y}_{k}\right), l, c\right] & \equiv\left(1-\bar{y}_{k}^{2}\right) F_{k}=0
\end{aligned}
$$

where $a_{r}=a\left(l \bar{x}_{r}+c\right)$. In the above equations the summation over the repeated index is implied. We are looking for the roots of the above system. We note that in above $r=1 \ldots(N+1)$ and together with the second equation we have total $(N+2)$ equations to solve for $(N+2)$ unknowns: $F_{1}, \ldots, F_{N}, l$ and $c$. The NewtonRaphson iteration is given by

$$
\begin{gathered}
{\left[\begin{array}{c}
F_{k} \\
l \\
c
\end{array}\right]_{n+1}=\left[\begin{array}{c}
F_{k} \\
l \\
c
\end{array}\right]_{n}-J_{n}^{-1}\left[\begin{array}{c}
N_{r} \\
R
\end{array}\right]_{n}} \\
J=\left[\begin{array}{lll}
\frac{\partial N_{r}}{\partial F_{k}} & \frac{\partial N_{r}}{\partial l} & \frac{\partial N_{r}}{\partial c} \\
\frac{\partial R}{\partial F_{k}} & \frac{\partial R}{\partial l} & \frac{\partial R}{\partial c}
\end{array}\right]_{(N+2) \times(N+1)}
\end{gathered}
$$

\section{References}

Dieterich, J. H., 1978. Time-dependent friction and the mechanics of stick-slip, Pure and Applied Geophysics, 116(4-5), 790-806.

Dieterich, J. H., 1979. Modeling of rock friction: 1. experimental results and constitutive equations, Journal of Geophysical Research: Solid Earth, 84(B5), 2161-2168.

Erdogan, F., Gupta, G. D., \& Cook, T. S., 1973. Numerical solution of singular integral equations, pp. 368-425, Springer Netherlands, Dordrecht.

Kato, N. \& Tullis, T. E., 2001. A composite rate- and state-dependent law for rock friction, Geophysical Research Letters, 28(6), 1103-1106.

Nagata, K., Nakatani, M., \& Yoshida, S., 2012. A revised rate-and state-dependent friction law obtained by constraining constitutive and evolution laws separately with laboratory data, Journal of Geophysical Research: Solid Earth, $117(\mathrm{~B} 2)$.

Rice, J. R. \& Ruina, A. L., 1983. Stability of steady frictional slipping, J. Appl. Mech., 50, 343-349.

Ruina, A., 1983. Slip instability and state variable friction laws, Journal of Geophysical Research, 88(B12), 10359-10370.

Tullis, T. E. \& Weeks, J. D., 1986. Constitutive behavior and stability of frictional sliding of granite, Pure and Applied Geophysics, 124(3), 383-414. 
Uenishi, K. \& Rice, J. R., 2003. Universal nucleation length for slip-weakening rupture instability under nonuniform fault loading, Journal of Geophysical Research: Solid Earth, 108(B1).

Viesca, R. C. \& Garagash, D. I., 2018. Numerical methods for coupled fracture problems, Journal of the Mechanics and Physics of Solids, 113, 13-34. 